\begin{tabular}{|c|c|}
\hline Title & A ssociations between cognitive impairment and quality of life in euthymic bipolar patients \\
\hline Author(s) & $\begin{array}{l}\text { Toyoshima, Kuniyoshi; Kako, Y uki; Toyomaki, A tsuhito; Shimizu, Y usuke; Tanaka, Teruaki; Nakagawa, Shin; Inoue, } \\
\text { Takeshi; Martinez-A ran, A nabel; V Vieta, Eduard; Kusumi, Ichiro }\end{array}$ \\
\hline Citation & $\begin{array}{l}\text { Psy chiatry research, } 271,510-515 \\
\text { https://doi .org/10.1016/.psychres.2018.11.061 }\end{array}$ \\
\hline Issue Date & $2019-01$ \\
\hline Doc URL & http:/hdl. handle.net/2115/76442 \\
\hline Rights & $\begin{array}{l}\text { (02018. This manuscript version is made available under the CC-BY-NC-ND } 4.0 \text { license } \\
\text { http://creativecommons.org/icenses/py-nc-nd/4.0/ }\end{array}$ \\
\hline Rights(URL) & http://reativecommons.org/icenses/by-nc-nd/4.0/ \\
\hline Type & article (author version) \\
\hline Additional Information & There are other files related to this item in HUSCAP. Check the above URL. \\
\hline File Information & 01_Psychiatry Res._271_510.pdf \\
\hline
\end{tabular}

Instructions for use 


\section{Associations between cognitive impairment and quality of life in euthymic bipolar patients}

Kuniyoshi Toyoshima ${ }^{\mathrm{a}, \mathrm{b}^{*}}$, Yuki Kako ${ }^{\mathrm{b}}$, Atsuhito Toyomaki ${ }^{\mathrm{b}}$, Yusuke Shimizuc, Teruaki Tanaka ${ }^{\mathrm{d}}$, Shin Nakagawa ${ }^{\mathrm{e}}$, Takeshi Inoue ${ }^{\mathrm{f}}$, Anabel Martinez-Aran ${ }^{\mathrm{g}}$, Eduard Vieta ${ }^{\mathrm{g}}$, Ichiro Kusumi ${ }^{\mathrm{b}}$

${ }^{a}$ Department of Psychiatry, Wakkanai City Hospital, Wakkanai, Japan

${ }^{\mathrm{b}}$ Department of Psychiatry, Graduate School of Medicine, Hokkaido University, Sapporo, Japan

${ }^{\mathrm{c}}$ Department of Psychiatry, Iwamizawa Municipal General Hospital, Iwamizawa, Japan

${ }^{\mathrm{d}}$ Department of Psychiatry, KKR Sapporo Medical Center, Sapporo, Japan

${ }^{\mathrm{e}}$ Division of Neuropsychiatry, Department of Neuroscience, Graduate School of Medicine, Yamaguchi University, Ube, Japan

${ }^{\mathrm{f}}$ Department of Psychiatry, Tokyo Medical University, Tokyo, Japan

${ }^{\mathrm{g}}$ Bipolar Disorders Program, Institute of Neurosciences, Hospital Clinic, University of Barcelona, IDIBAPS, CIBERSAM. Villarroel 170, Barcelona, 08036 Catalonia, Spain

\section{Corresponding Author}

Kuniyoshi Toyoshima, MD, Ph.D., Department of Psychiatry, Wakkanai City Hospital, 3-13-15, Chuo, Wakkanai-shi, Hokkaido 097-8555, Japan

Tel: 0162232771

Fax: 0162235636

E-mail: toyoshima@med.hokudai.ac.jp 


\section{Associations between cognitive impairment and quality of life in euthymic bipolar patients}

\section{ABSTRACT}

During the euthymic state in bipolar disorder, cognitive functions often remain affected. Specifically, subjective and objective cognitive impairment might distinctly affect patients’ quality of life (QoL); however, this question had not been examined previously in Japanese patients. Therefore, the current study investigated the associations between cognitive complaints, QoL, and objective cognitive functions. Forty patients in remission were recruited from the Hokkaido University Hospital, Sapporo, Japan and assessed with the translated version of the cognitive complaints in bipolar disorder rating assessment (COBRA), medical outcomes study 36-item short-form health survey version 2 (SF-36v2), and Sheehan disability scale (SDS). The Japanese adult reading scale, Wisconsin card sorting test, word fluency, continuous performance test, trail making test (TMT), auditory verbal learning, and Stroop test evaluated objective cognitive functions. Significant correlations were observed between the COBRA, SF-36v2, and SDS results, as well as the TMT scores. Overall, euthymic patients were aware of their cognitive dysfunction, which could be understood in relation to the decrease in satisfaction in their daily life. Therefore, even mild cognitive impairments can have ramifications for patients in the euthymic state of bipolar disorder.

Keywords: Bipolar disorder; cognitive complaints; cognitive function; neuropsychological assessment; COBRA; SF-36v2; SDS 


\section{Introduction}

Bipolar disorder is characterized by recurring episodes of manic and depressive states according to the Diagnostic and Statistical Manual of Psychiatric Disorders, fifth version (DSM-5) (American Psychiatric Association, 2013). During mania and depression epochs, bipolar patients show deficits in various cognitive domains (Martínez-Arán et al., 2004a; Porter et al., 2015), which can be explained by severe mood changes (Basso et al., 2002; Glahn et al., 2007). Furthermore, some patients still experience cognitive impairment during euthymic epochs that can affect attention, executive function, and memory (Basso et al., 2002; Joe et al., 2008; Martínez-Arán et al. 2004b; Sumiyoshi et al., 2017), suggesting that cognitive dysfunction is not merely caused by the extreme mood during manic or depressed phases.

Miskowiak et al. (2018) reported heterogeneity among remitted bipolar patients: 12$40 \%$ of patients have global cognitive impairments across several domains, 29-40\% show selective deficits in attention and psychomotor speed, whereas 32-48\% are cognitively intact compared to normal controls. Therefore, more than half of the patients in remission experience some form of cognitive dysfunction, which is concordance with previous reports (Burdick et al., 2015; Cullen et al., 2016). These impairments negatively impact abilities and activities relevant to daily living (e.g., work-related performance, paying bills, and maintaining social relations) and can severely affect the quality of life (QoL) of bipolar patients. In a meta-analysis, Michalak et al. (2005) evaluated several studies assessing the relationship between bipolar disorder and QoL and emphasized that non-symptomatic, euthymic patients are less prone to the effects of cognitive distortion; thus, the measured effects on QoL are less affected by such distortions.

A wide range of instruments has been used to assess neurocognitive functions, which were divided into subjective and objective measures (Demant et al., 2015; Jensen et al., 
2015). To accurately diagnose subjective cognitive impairments, a self-reported instrument, i.e., the cognitive complaints in bipolar disorder rating assessment (COBRA), was recently developed (Rosa et al., 2013), and was used in various studies on cognitive impairment and QoL (Jensen et al., 2015). Relatively few studies have examined the relationship between cognitive impairment evaluated with the COBRA and QoL measurements that include both SF-36 and SDS. Bipolar and mood disorders have been studied predominantly in Western countries (North America and Europe); therefore, a Japanese version was developed, validated, and applied in bipolar patients (Toyoshima et al., 2017). The current study further elucidated the relationship between cognitive impairment, as assessed with the COBRA, and QoL in euthymic patients with bipolar disorder in a Japanese sample.

Although cognitive impairment is generally mild in bipolar patients, a study suggested an association between compromised prefrontal functions and illness awareness (Shad et al., 2015), which can have further implications for medication compliance. Few studies have investigated the relationship between patients' awareness of illness and their subjective and objective cognitive impairment in the remission phase of bipolar disorder. Therefore, cognitive impairments should be explored according to the recommendations of the International Society for Bipolar Disorders Cognition Task Force (Miskowiak et al., 2017). Subjective and objective cognitive functions might be differentially affected in euthymic bipolar patients, which in turn could have distinct effects on QoL. However, such research has not yet been conducted in Japanese patients and was instead further explored in the current study.

\section{Methods}

\subsection{Participants}

All participants were recruited through convenience sampling between September 2014 and 
August 2015 from the Hokkaido University Hospital, Sapporo, Japan. Among the 40 patients included in this study, 13 were diagnosed with bipolar subtype I and 27 with subtype II. The diagnosis was made by an attending psychiatrist according to the DSM-5 criteria (American Psychiatric Association, 2013). These patients satisfied the remission criteria $[\leq 7$ points on both the Hamilton rating scale for depression (17-HAM-D) and the young mania rating scale (YMRS) for at least 8 weeks before the assessment]. The diagnosis was subsequently discussed and confirmed by other psychiatrists. Other inclusion criteria were an age range of 18-64 years and being an outpatient at the Department of Psychiatry, Hokkaido University Hospital. The current study was approved by the Local Ethics Committee of Hokkaido University (Ethics Approval Number: 014-0006). After the explanation of the complete study, all subjects provided written informed consent to participate.

\subsection{Assessments}

The tests for subjective cognitive function (COBRA), QoL (SF-36 and SDS), and neuropsychological assessment were completed in 60-90 min with regular breaks.

\subsubsection{Clinical and sociodemographic assessment}

Socio-demographic and clinical data were collected from the electronic patient charts available at the participating hospital (Table 1). Patient medication information was recorded (Table 2) but not included as a control factor. The 17-HAM-D and YMRS were administered to assess depressive and manic symptoms, respectively. The intelligence level was assessed with the Japanese adult reading scale-25-word version (JART-25, Matsuoka et al., 2006), which represents an estimate of pre-illness intelligence.

\subsubsection{Subjective cognitive measures}


The COBRA is a 16-item self-reported instrument for measuring subjective cognitive dysfunction, including executive function, processing speed, working memory, verbal learning and memory, attention/concentration, and mental tracking (Rosa et al., 2013). All items are rated using a 4 -point scale $(0=$ never, $1=$ sometimes, $2=$ often, and $3=$ always $)$. The questions are based on everyday mental abilities. The total COBRA score is calculated by summing the ratings for each item; the maximum score is 48 , with higher scores indicating higher levels of subjective complaints.

The original Spanish version of the COBRA was developed by the Bipolar Disorder Program at the Hospital Clinic of Barcelona to assess cognitive complaints (Rosa et al., 2013). Successively, it was translated into Japanese and a back-translation was approved by the original authors (Toyoshima et al., 2017) to ensure that the translated version remained unaltered. The International Society for Bipolar Disorders Targeting Cognition Task Force recommends the COBRA for screening, in addition to an objective cognitive measure in a clinical context (Miskowiak et al., 2018).

\subsubsection{QoL assessment}

The Medical Outcomes Study 36-Item Short-Form Health Survey Version 2 (SF-36v2) addresses health-related aspects of QoL (McHorney et al., 1994), including physical functioning, bodily pain, general and mental health, and vitality, and has been applied in the examination of QoL in bipolar patients (Anaya et al., 2016; Bonnín et al., 2012; GutiérrezRojas et al., 2008). It is a self-assessment questionnaire, which patients can complete at home and return to the physician at the next appointment. The 36 items cover eight dimensions that can be classified into two main components. The physical component summary (PCS) includes physical functioning (PF, 10 items); role-physical functioning, namely limitations attributed to physical problems (RP, 4 items); bodily pain (BP, 2 items); and general health 
perception (GH, 5 items). In contrast, the mental component summary (MCS) comprises vitality (VT; 4 items); social functioning (SF, 2 items); role-emotional, namely limitations attributed to emotional problems (RE, 3 items); and mental health (MH, 5 items). One item asks about changes in the health status. Scores are transformed into 0 (worst possible health) to 100 (best health); where lower scores indicate poorer health or greater disability. A recent Japanese study (Suzukamo et al., 2011) developed a three-component model, with the role component summary (RCS) as a third factor, that was strongly correlated with RP, SF, and RE. This three-component model was applied to the current sample.

The Japanese version of the SF-36v2 was developed and validated for the general population (Fukuhara and Suzukamo, 2004; Fukuhara et al., 1998a; Fukuhara et al., 1998b). A national Japanese norm was established, for which the mean and SD of the patient's (0100) scoring of each domain can be transformed to a norm-based score (NBS) using the following equation: NBS $=10 \times[(0-100$ score $-\mathrm{ASNN}) /(\mathrm{SD}$ of ASNN $)]+50($ Fukuhara et al., 2007), where ASNN represents the age- and sex-stratified national norm. Such normbased scores (mean $=50, \mathrm{SD}=10$ ), rather than the $0-100$ scoring, are recommended (Ware et al., 2007).

\subsubsection{Disability and impairment measures}

The Sheehan disability scale (SDS) (Sheehan et al., 1996) measures global impairment and includes three items on impairment affecting work, social, and family life, representing additional aspects of QoL. This scale has been used to study bipolar patients (Arbuckle et al. 2009; Endicott et al., 2008). The patient rates the extent to which work/school, social life, and family life/home responsibilities are impaired by his or her symptoms on a 10-point visual analog scale ranging from 0 (no impairment), 1-3 (mild impairment), 4-6 (moderate impairment), 7-9 (marked impairment), to 10 (extreme disability). Each of the three items is 
introduced with a question: "Have the symptoms disrupted your work/school, social life, and family life/home responsibilities?” The total scores range from 0 to 30, with higher scores indicating greater disruption in the three domains due to mental illness (Sheehan et al., 2011).

\subsubsection{Neuropsychological assessment}

Neuropsychological function (i.e., objective cognitive function) was assessed by attending neuropsychologists using tests from the cognitive function assessment battery at the Hokkaido University Hospital Department of Psychiatry (Toyomaki et al., 2008): 1) The Wisconsin card sorting test (WCST) measures the number of categories achieved (WCSTCA) and perseverative errors (PEM, Milner type) and evaluates executive function; 2) The word fluency test (WFT) assesses verbal fluency and the aspects of executive control; 3) The continuous performance test examines sustained attention (CPT errors) and reaction time (CPTRT); 4) the trail making test (TMT-A, connecting numerical sequence; TMT-B, connecting alternating numeric and alphabetic sequence) assesses visual processing and movement speed, as well as executive function; 5) the auditory verbal learning test evaluates instant (AVLTi) and delayed recall (AVLTr); 6) the Stroop test (ST) contains 24 dots printed in different colors or 24 color words; each printed in a different, non-congruent color. Patients are instructed to name the print color of the dots and words as quickly as possible. The number of errors (STerr) and reaction time difference (STRT) between naming color of dots and words reflect response suppression and selective attention.

\subsection{Statistical analyses}

Spearman correlations assessed the relationships between COBRA, QoL (SDS, 3 items; SF36v2, 8 domains), and objective cognitive functions (neuropsychological tests). Partial correlations, with the 17-HAM-D score as the control variable, are presented in 
Supplementary Table 1. Linear regressions quantified the associations between COBRA and SDS and between COBRA and TMT-A. All statistical analyses were performed with SPSS Version 23.0; $p<0.05$ was considered statistically significant.

\section{Results}

The socio-demographic and clinical characteristics of the 40 patients are summarized in Table 1. A slightly higher number of female patients participated, and about two-thirds of all patients were diagnosed with bipolar II subtype. The average COBRA score (13.68 \pm 8.04$)$

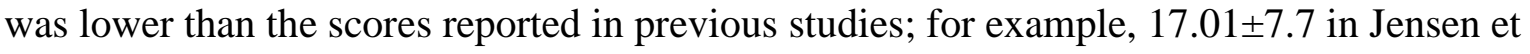
al. (2015) and 16.7 \pm 9.8 in Rosa et al. (2013).

The average SF-36v2 scores were 47.2 \pm 11.32 for PCS, $45.44 \pm 9.91$ for MCS, and $35.01 \pm 17.49$ for RCS and were comparable to or lower than those reported in other studies; for example, 49.5 for PCS and 38.0 for MCS in Anaya et al. (2016) and 51.1 for PCS and 43.0 for MCS in Bonnín et al. (2012). When compared to the study by Suzukamo et al. (2011), the average scores for the three components were lower in the current study, suggesting that these patients experienced greater disability and inferior health. The total SDS score was 9.03, indicating mild to moderate disability. This average was lower than that reported for euthymic bipolar patients in other studies; for example, 15.8 in Arbuckle et al. (2009). Therefore, the current patients presented comparatively less disability in the three domains of work/school, social life, and family/home.

\subsection{Association between subjective cognitive impairment and QoL}

Direct and partial correlation analyses (Table 3 and Supplementary Table 1, respectively) confirmed the significant associations between the COBRA total score and the majority of the QoL scores. The correlation between COBRA and 17-HAM-D scores was significant (Table 
3), which would require controlling for depressive symptoms. However, even when controlling for depressive symptoms using the 17-HAM-D scores, the correlations pattern remained very similar. The COBRA score was positively correlated with SDS total (Figure 1), as further confirmed by the linear regression $(F=9.795, p=.003)$. In contrast, it was negatively correlated with all the sub-scores and the two component summaries (MCS and RCS) of the SF-36v2 (Table 3). Lower scores, suggesting greater disability, were therefore associated with higher levels of subjective cognitive complaints. This was particularly the case for social, emotional, and mental health domains, which showed the highest negative correlations ( $r s<-.58$ ). Therefore, both the QoL measures revealed that higher subjective cognitive impairments were related to greater disruptions in daily life. Among the various QoL sub-scores, the SDS social showed strong negative correlations with most domains of the SF-36v2 (PF, RP, GH, VT, SF, RE, and MH; with PF, SF, MH rs < -.46), confirming their validity.

\subsection{Association between subjective cognitive impairment and objective cognitive function}

The correlation and regression analyses (Table 4, Figure 2) revealed that the COBRA total score was significantly correlated only with the TMT-A $(F=11.536, p=.002)$. Therefore, decreased processing speed (i.e., longer time in completing the test) was positively associated with greater subjective cognitive dysfunction. Average scores on the various neuropsychological tests (including range and SD) are presented in Supplementary Table 2.

\subsection{Association between objective cognitive function and $Q o L$}

Several objective cognitive tests showed significant correlations with QoL measurements. SDS family/home was negatively correlated with the STerr $(r=-.38, p=.016)$

(Supplementary Table 3). TMT-A yielded multiple negative correlations with four sub-scores 
of the SF-36v2 (RE $r=-.419, p=.007$; MH $r=-.373, p=.018$; PCS $r=-.402, p=.001$ ), STRT was negatively correlated with PF ( $r=-.394, p=.012)$, while TMT-B was correlated with two SF-36v2 sub-scores (MH $r=-.354, p<.025$; PCS $r=-.447, p=.004$ ). AVLTi was positively correlated with two SF-36v2 sub-scores ( $\mathrm{MH} r=-.357, p<.024$; RP $r=-.386, p$ $=.014)$. Overall, longer processing times and impaired memory were related to lower QoL, indicative of greater disability.

\subsection{Association between objective cognitive function and clinical parameters}

The total scores of the two clinical rating scales for manic (YMRS) and depressive (17HAM-D) symptoms were significantly correlated with the CPT error $(r=.384, p<.05)$ and the CPTRT $(r=.529, p<.01)$, respectively, suggesting that the severity of mood disorders affected sustained attention and reaction time. The 17-HAM-D score was positively associated with the TMTA $(r=.402, p<.05)$, confirming that depression interfered with visual processing and mental flexibility.

\section{Discussion}

The present study examined the associations between subjective cognitive impairment, QoL, and objective cognitive functioning in a group of Japanese patients who were in remission from bipolar disorder by using the recently developed Japanese version of the COBRA (Toyoshima et al., 2017). Compared to other studies, patients in the current study showed milder subjective cognitive dysfunction, possibly given the relatively strict current criteria for defining euthymic patients, whereas other studies included patients during various phases of the disorder. However, even if such patients do not manifest typical extreme moods, they still reportedly have cognitive dysfunctions (Anaya et al., 2016; Bora et al., 2013; Demant et al., 2015; Martínez-Arán et al., 2004a). 
Objective cognitive functions in the current study were found to be impaired in some domains (e.g., TMT-A, TMT-B), suggesting overall slower processing speed. Both subjective and objective impairments, which mainly affect memory (Sumiyoshi et al., 2017) and executive function, can worsen the course and outcome of the disorder by disrupting psychosocial adaptation and adherence to treatment. For example, Jensen et al. (2015) confirmed strong associations between the COBRA and the measures of socio-occupational function. Although cognitive impairments are subtle and subjective, it is important to identify them at an early stage to implement treatment strategies. Therefore, the availability of simple, reliable, and valid tools, including the COBRA, to assess these impairments is crucial.

The present study further reported that these subjective cognitive impairments were significantly correlated with self-reported measures of QoL after controlling for the level of depressive symptoms. Seven of the eight domains of the SF-36v2 yielded significant negative correlations with the COBRA, suggesting that bipolar patients were aware of their own cognitive dysfunction, which could be understood in relation to the decrease in satisfaction in their daily life. QoL was not just correlated with subjective but also with several measures of objective cognitive functioning, pertaining mostly to processing speed, memory, and attention.

Patients with fewer subjective cognitive dysfunctions were also more attentive and therefore more aware of how they responded to their medication. In contrast, patients with greater subjective cognitive dysfunction might be easily distracted and less able to adhere to a strict medication schedule. Consequently, a lapse in medication adherence could further diminish cognitive abilities (subjective and objective), which highlights the importance of improving awareness of mental illness, not just in cases of schizophrenia, but also in cases involving bipolar disorder. Cognitive dysfunction could impair daily living activities, as suggested by Träger et al. (2017) who showed significant correlations between processing 
speed and activities of daily living (ADL), while the COBRA scores were not correlated with the ADL. Since considerable cognitive heterogeneity in euthymic patients exists, it would seem reasonable to examine them according to their distinct scores. For example, Burdick et al. (2014) reported three distinct neurocognitive sub-groups in bipolar disorder. This means increasing the number of cases in future studies and examining subjective cognitive complaints by subgroups.

When assessing subjective cognitive impairments, a main issue is how well they correlate with objectively measured cognitive dysfunctions. Demant et al. (2015) reported weak but significant correlations between objective and subjective measures of cognitive functions. As objective cognitive and psychosocial functioning were not correlated, subjective cognitive complaints were more likely to reflect residual depressive symptoms and not actual cognitive impairments (Demant et al., 2015; Porter et al., 2015). The current study partially supports this observation in that the COBRA was only correlated with one neuropsychological test (TMTA). Contrastingly, residual effects of depression were accounted for in the present investigation by computing partial correlations, suggesting that lingering depressive symptoms could not solely explain the subjective cognitive complaints.

\subsection{Limitations}

Considering that the present investigation was an exploratory study, correction of multiple comparisons was not performed. The present sample size was relatively small and various important factors, including medication or socio-demographic characteristics, were not controlled for. Although correlations were highly significant for many relevant assessments, the cross-sectional design precludes a causal interpretation of these relationships. Therefore, whether subjective cognitive complaints contributed to reduced QoL or objective cognitive impairments led to subjective complaints could not be determined. Further, possible effects of 
medication, smoking, alcohol use, and physical health on cognition were not included. The associations between the COBRA, QoL, and objective neuropsychological functions should be further investigated in longitudinal studies using larger patient sample sizes and more stringent control of confounding variables. Comparisons with non-clinical populations or patients with other psychiatric disorders should be performed to establish the correlations profiles across clinical and non-clinical spectra. According to Burdick et al. (2015), functional outcome measures should be sensitive enough to capture disability. The SF-36v2 and SDS rely on patients' self-reports and therefore might not be as accurate. These subjective assessments should be accompanied by objective information (e.g., observations, informants) whenever possible.

\subsection{Conclusions}

Subjective cognitive impairments should be considered important indicators and mediators of psychosocial functioning in euthymic patients with bipolar disorder. Crucial issues, including medication adherence and insights into the disorder, are affected by cognitive functioning. It is therefore important to assess these factors in combination with objective measurements of cognition, psychosocial functioning, and adaptation. The current study supports the conclusion that stronger subjective cognitive impairments are related to lower estimates of QoL, indicating overall poorer functioning in various daily life realms.

Declarations of interest: The authors declare no conflicts of interest.

Acknowledgements: The authors thank Ms. Adriane R. Rosa and her colleagues in the Bipolar Disorders Program, Institute of Neurosciences, Hospital Clinic, University of Barcelona and Mr. Morihiro Musha of the Musha-Clinic. Editorial support was provided by 
Editage.

Funding: This research did not receive any specific grant from funding agencies in the public, commercial, or non-profit sectors. 


\section{References}

American Psychiatric Association, 2013. Diagnostic and Statistical Manual of Psychiatric Disorders, fifth ed. American Psychiatric Association, Washington DC.

Anaya, C., Torrent, C., Caballero, F.F., Vieta, E., Bonnin, C.M., Ayuso-Mateos, J.L., CIBERSAM Functional Remediation Group, 2016. Cognitive reserve in bipolar disorder: relation to cognition, psychosocial functioning and QoL. Acta Psychiatr. Scand. 133, 386-398. doi: http://dx.doi.org/10.1111/acps.12535.

Arbuckle, R., Frye, M.A., Brecher, M., Paulsson, B., Rajagopalan, K., Palmer, S., Degl'Innocenti, A., 2009. The psychometric validation of the Sheehan Disability Scale (SDS) in patients with bipolar disorder. Psychiatry Res. 165, 163-174. doi: http://dx.doi.org/10.1016/j.psychres.2007.11.018.

Basso, M.R., Lowery, N., Neel, J., Purdie, R., Bornstein, R.A., 2002. Neuropsychological impairment among manic, depressed, and mixed-episode inpatients with bipolar disorder. Neuropsychology 16, 84-91.

Bonnín, C.M., Sanchez-Moreno, J., Martinez-Aran, A., Sole, B., Reinares, M., Rosa, A.R., Goikolea, J.M., Benabarre, A., Ayuso-Mateos, J.L., Ferrer, M., Vieta, E., 2012. Subthreshold symptoms in bipolar disorder: Impact on neurocognition, QoL and disability. J. Affect. Disord. 136, 650-659. doi: http://dx.doi.org/10.1016/j.jad.2011.10.012.

Bora, E., Harrison, B.J., Yucel, M., Pantelis, C., 2013. Cognitive impairment in euthymic major depressive disorder: a meta-analysis. Psychol. Med. 43, 2017-2026. doi: http://dx.doi.org/10.1017/s0033291712002085.

Burdick, K. E., Ketter, T. A., Goldberg, J. F., \& Calabrese, J. R., 2015. Assessing Cognitive Function in Bipolar Disorder: Challenges and Recommendations for Clinical Trial Design. J. Clin. Psychiatry 76, e342-e350. doi: 
http://dx.doi.org/10.4088/JCP.14cs09399.

Burdick, K. E., Russo, M., Frangou, S., Mahon, K., Braga, R. J., Shanahan, M., \& Malhotra, A. K., 2014. Empirical evidence for discrete neurocognitive subgroups in bipolar disorder: clinical implications. Psychol. Med. 44, 3083-3096. doi: http://dx.doi.org/10.1017/s0033291714000439.

Cullen, B., Ward, J., Graham, N. A., Deary, I. J., Pell, J. P., Smith, D. J., \& Evans, J. J., 2016. Prevalence and correlates of cognitive impairment in euthymic adults with bipolar disorder: A systematic review. J. Affect. Disord. 205, 165-181. doi: http://dx.doi.org/10.1016/j.jad.2016.06.063.

Demant, K.M., Vinberg, M., Kessing, L.V., Miskowiak, K.W., 2015. Assessment of subjective and objective cognitive function in bipolar disorder: correlations, predictors and the relation to psychosocial function. Psychiatry Res. 229, 565-571. doi: http://dx.doi.org/10.1016/j.psychres.2015.05.022.

Endicott, J., Paulsson, B., Gustafsson, U., Schiöler, H., Hassan, M., 2008. Quetiapine monotherapy in the treatment of depressive episodes of bipolar I and II disorder: Improvements in QoL and quality of sleep. J. Affect. Disord. 111, 306-319. doi: http://dx.doi.org/10.1016/j.jad.2008.06.019.

Fukuhara, S., Bito, S., Green, J., Hsiao, A., Kurokawa, K., 1998a. Translation, adaptation, and validation of the SF-36 Health Survey for use in Japan. J. Clin. Epidemiol. 51, 1037-1044. doi: https://doi.org/10.1016/S0895-4356(98)00095-X.

Fukuhara, S., Suzukamo Y., 2004. Manual of SF-36v2 Japanese version. Institute for Health Outcomes \& Process Evaluation research, Kyoto, Japan.

Fukuhara, S., Ware, J.E., Kosinski, M., Wada, S., Gandek, B., 1998b. Psychometric and clinical tests of validity of the Japanese SF-36 Health Survey. J. Clin. Epidemiol. 51, 1045-1053. doi: https://doi.org/10.1016/S0895-4356(98)00096-1. 
Fukuhara, S., Yamazaki S., Marumo F., Akiba T., Akizawa T., Fujimi S., Haruki, S., Kawaguchi, Y., Nihei, H., Shoji, T., Maeda, T., 2007. Health-related QoL of predialysis patients with chronic renal failure. Nephron Clin. Pract. 105, c1-c8.

Glahn, D.C., Bearden, C.E., Barguil, M., Barrett, J., Reichenberg, A., Bowden, C.L., Soares, J.C., Velligan, D.I., 2007. The neurocognitive signature of psychotic bipolar disorder. Biol. Psychiatry 62, 910-916. doi: http://dx.doi.org/10.1016/j.biopsych.2007.02.001. Gutiérrez-Rojas, L., Gurpegui, M., Ayuso-Mateos, J.L., Gutiérrez-Ariza, J.A., Ruiz-Veguilla, M., Jurado, D., 2008. QoL in bipolar disorder patients: a comparison with a general population sample. Bipolar Disord. 10, 625-634. doi: http://dx.doi.org/10.1111/j.1399-5618.2008.00604.x.

Jensen, J.H., Støttrup, M.M., Nayberg, E., Knorr, U., Ullum, H., Purdon, S.E., Kessing, L.V., Miskowiak, K.W., 2015. Optimising screening for cognitive dysfunction in bipolar disorder: validation and evaluation of objective and subjective tools. J. Affect. Disord. 187, 10-19. doi: http://dx.doi.org/10.1016/j.jad.2015.07.039.

Joe, S., Joo, Y., Kim, S., 2008. Experience of subjective symptoms in euthymic patients with bipolar disorder. J. Korean Med. Sci. 23, 18-23.

Martínez-Arán, A., Vieta, E., Colom, F., Torrent, C., Sánchez-Moreno, J., Reinares, M., Benabarre, A., Goikolea, J.M., Brugue, E., Daban, C., Salamero, M., 2004a. Cognitive impairment in euthymic bipolar patients: implications for clinical and functional outcome. Bipolar Disord. 6, 224-232. doi: http://dx.doi.org/10.1111/j.13995618.2004.00111.x.

Martínez-Arán A., Vieta, E., Reinares, M., Colom, F., Torrent, C., Sánchez-Moreno, J., Benabarre, A., Goikolea, J.M., Comes, M., Salamero, M., 2004b. Cognitive function across manic or hypomanic, depressed, and euthymic states in bipolar disorder. Am. J. Psychiatry 161, 262-270. doi: http://dx.doi.org/10.1176/appi.ajp.161.2.262. 
Matsuoka, K., Uno, M., Kasai, K., Koyama, K., Kim, Y., 2006. Estimation of premorbid IQ in individuals with Alzheimer’s disease using Japanese ideographic script (Kanji) compound words: Japanese version of National Adult Reading Test. Psychiatry Clin. Neurosci. 60, 332-339. doi: http://dx.doi.org/10.1111/j.1440-1819.2006.01510.x. McHorney, C.A., Ware, J.E., Lu, J.F.R., Sherbourne, C.D., 1994. The MOS 36-item shortform health Survey (SF-36): III. tests of data quality, scaling assumptions, and reliability across diverse patient groups. Med. Care. 32, 40-66.

Michalak, E.E., Yatham, L.N., Lam, R.W., 2005. QoL in bipolar disorder: a review of the literature. Health Qual. Life Outcomes 3, 1-17. doi: http://dx.doi.org/10.1186/14777525-3-72.

Miskowiak, K.W., Burdick, K.E., Martinez-Aran, A., Bonnin, C.M., Bowie, C.R., Carvalho, A.F., Gallagher, P., Lafer, B., López-Jaramillo, C., Sumiyoshi, T., McIntyre, R.S., Schaffer, A., Porter, R.J., Torres, I.J., Yatham, L.N., Young, A.H., Kessing, L.V., Vieta, E., 2017. Methodological recommendations for cognition trials in bipolar disorder by the International Society for Bipolar Disorders Targeting Cognition Task Force. Bipolar Disord. 19, 614-626. doi: https://doi.org/10.1111/bdi.12534.

Miskowiak, K.W., Burdick, K.E., Martinez-Aran, A., Bonnin, C.M., Bowie, C.R., Carvalho, A.F., Gallagher, P., Lafer, B., López-Jaramillo, C., Sumiyoshi, T., McIntyre, R.S., Schaffer, A., Porter, R.J., Purdon, S., Torres, I.J., Yatham, L.N., Young, A.H., Kessing, L.V., Vieta, E., 2018. Assessing and addressing cognitive impairment in bipolar disorder: the International Society for Bipolar Disorders Targeting Cognition Task Force recommendations for clinicians. Bipolar Disord. 20, 184-194. https://doi.org/10.1111/bdi.12595

Porter, R.J., Robinson, L.J., Malhi, G.S., Gallagher, P., 2015. The neurocognitive profile of mood disorders - a review of the evidence and methodological issues. Bipolar Disord. 
17, 21-40. doi: https://doi.org/10.1111/bdi.12342.

Rosa, A.R., Mercadé, C., Sánchez-Moreno, J., Solé, B., Mar Bonnin, C.D., Torrent, C., Grande, I., Sugranyes, G., Popovic, D., Salamero, M., Kapczinski, F., Vieta, E., Martinez-Aran, A., 2013. Validity and reliability of a rating scale on subjective cognitive deficits in bipolar disorder (COBRA). J. Affect. Disord. 150, 29-36. doi: https://doi.org/10.1016/j.jad.2013.02.022.

Shad, M.U., Prasad, K., Forman, S.D., Haas, G.L., Walker, J.D., Pisarov, L.A., Goldstein, G., 2015. Insight and neurocognitive functioning in bipolar subjects. Compr. Psychiatry 56, 112-120. doi: http://dx.doi.org/10.1016/j.comppsych.2014.04.016.

Sheehan, D.V., Harnett-Sheehan, K., Raj, B.A., 1996. The measurement of disability. Int. Clin. Psychopharmacol. 11, 89-95.

Sheehan, D.V., Harnett-Sheehan, K., Spann, M.E., Thompson, H.F., Prakash, A., 2011. Assessing remission in major depressive disorder and generalized anxiety disorder clinical trials with the discan metric of the Sheehan disability scale. Int. Clin. Psychopharmacol. 26, 75-83. doi: http://dx.doi.org/10.1097/YIC.0b013e328341bb5f. Sumiyoshi, T., Toyomaki, A., Kawano, N., Kitajima, T., Kusumi, I., Ozaki, N., Iwata, N., Sueyoshi, K., Nakagome, K., 2017. Verbal memory impairment in patients with subsyndromal bipolar disorder. Front. Psychiatry 8, 168. doi: http://dx.doi.org/10.3389/fpsyt.2017.00168.

Suzukamo, Y., Fukuhara, S., Green, J., Kosinski, M., Gandek, B., Ware, J.E., 2011. Validation testing of a three-component model of Short Form-36 scores. J. Clin. Epidemiol. 64, 301-308. doi: http://dx.doi.org/10.1016/j.jclinepi.2010.04.017.

Toyomaki, A., Kusumi, I., Matsuyama, T., Kako, Y., Ito, K., Koyama, T., 2008. Tone duration mismatch negativity deficits predict impairment of executive function in schizophrenia. Prog. Neuropsychopharmacol. Biol. Psychiatry 32, 95-99. doi: 
https://doi.org/10.1016/j.pnpbp.2007.07.020.

Toyoshima, K., Fujii, Y., Mitsui, N., Kako, Y., Asakura, S., Martinez-Aran, A., Vieta, E., Kusumi, I., 2017. Validity and reliability of the Cognitive Complaints in Bipolar Disorder Rating Assessment (COBRA) in Japanese patients with bipolar disorder. Psychiatry Res. 254, 8-89. doi: https://doi.org/10.1016/j.psychres.2017.04.043.

Träger, C., Decker, L., Wæhrens, E. E., Knorr, U., Miskowiak, K., \& Vinberg, M., 2017. Influences of patient informed cognitive complaints on activities of daily living in patients with bipolar disorder. An exploratory cross-sectional study. Psychiatry Res. 249, 268-274. doi: https://doi.org/10.1016/j.psychres.2016.12.058.

Ware, J., Kosinski, M., Bjorner, J., Turner-Bowker, D., Gandek, B., \& Maruish, M. E., 2007. User's Manual for the SF-36v2® Health Survey. Lincoln, RI: Quality Metric. 


\section{Figure legends}

Figure 1. Correlation between the Sheehan disability scale (SDS) total score and the cognitive complaints in bipolar disorder rating assessment (COBRA) total score.

Figure 2. Correlation between the trail making test (numerical sequence, TMT-A) score (in seconds) and the cognitive complaints in bipolar disorder rating assessment (COBRA) total score. 


\section{Tables}

Table 1. Clinical and socio-demographic characteristics $(\mathrm{N}=40)$.

Patient characteristics $\quad$ Mean (SD), n (\%)

IQ (JART-25), mean (SD)

$107(10.28)$

Illness duration, mean (SD)

$15.75(8.8)$

Age, mean (SD)

$43.63(10.48)$

Age at onset, mean (SD)

$28.05(8.88)$

Sex male, $n(\%)$

$18(45.0)$

Years of education, mean (SD)

$14.33(2.27)$

Depressive onset, mean (SD)

$27.15(10.44)$

Number of hospitalizations, mean (SD)

$1.78(2.2)$

Number of total episodes, mean (SD)

$5.7(3.77)$

Number of hypomanic episodes, mean (SD)

Number of manic episodes, mean (SD)

$0.78(1.42)$

Number of depressive episodes, mean (SD)

Number of mixed episodes, mean (SD)

$0.3(0.56)$

Number of suicide attempts, mean (SD)

$0.5(0.96)$

17-HAM-D score, mean (SD)

YMRS score, mean (SD)

$0.33(1.00)$

Abbreviations: JART-25, Japanese adult reading scale-25-word version; 17-HAM-D, 
Hamilton rating scale for depression; YMRS, young mania rating scale. 
Table 2. Patients' medications $(\mathrm{N}=40)$

\begin{tabular}{|c|c|c|c|}
\hline Medication name/class & Max (mg) & Average (mg) & $\mathrm{SD}(\mathrm{mg})$ \\
\hline $\mathrm{Li}$ & 1200 & 410 & 371.276 \\
\hline VPA & 1600 & 270 & 428.593 \\
\hline CBZ & 500 & 28.75 & 106.149 \\
\hline LTG & 400 & 116.88 & 151 \\
\hline CP conversion & 826 & 190.375 & 225.267 \\
\hline IMP conversion & 150 & 13.438 & 36.404 \\
\hline DZP conversion (anxiolytic) & 80 & 10.1 & 19.515 \\
\hline Total DZP conversion (anxiolytic and hypnotic) & 80 & 15.56 & 20.742 \\
\hline $\begin{array}{l}\text { Abbreviations: Li, lithium; VPA, valproate; CBZ, } \\
\text { chlorpromazine; IMP, imipramine; DZP diazepam }\end{array}$ & carbamazep & ne; LTG, lamot & gine; CP, \\
\hline
\end{tabular}


Table 3. Spearman correlations between the COBRA, 17-HAM-D, and QoL measures $(\mathrm{N}=40)$.

\begin{tabular}{|c|c|}
\hline & COBRA \\
\hline 17-HAMD-D & $.352 *$ \\
\hline SDS work & $.375 *$ \\
\hline SDS social & $.568 * *$ \\
\hline SDS & $.336 *$ \\
\hline \multicolumn{2}{|l|}{ family/home } \\
\hline SDS total & $.498 * *$ \\
\hline PF_N & $-.367^{*}$ \\
\hline RP_N & $-.446 * *$ \\
\hline BP_N & $-.341^{*}$ \\
\hline GH_N & $-.407 * *$ \\
\hline VT_N & $-.538 * *$ \\
\hline SF_N & $-.634 * *$ \\
\hline RE_N & $-.665 * *$ \\
\hline MH_N & $-.587 * *$ \\
\hline PCS & -.156 \\
\hline MCS & $-.329 *$ \\
\hline RCS & $-.595 * *$ \\
\hline
\end{tabular}

Abbreviations: COBRA, cognitive complaints in bipolar disorder rating assessment; 17-HAM-D, Hamilton rating scale for depression; SDS, Sheehan disability scale; SF-36, Medical Outcomes Study-Short Form Health Survey; PF, physical functions; RP, role—physical; BP, bodily pain; GH, general health; VT, vitality; SF, social functioning; RE, role-emotional; MH, mental health; PCS, 
physical component summary; MCS, mental component summary; RCS, role component summary. ${ }^{*} \mathrm{p}<.05,{ }^{* *} \mathrm{p}<.01$ (two-sided). 
Table 4. Spearman correlations between the COBRA and neuropsychological measures $(\mathrm{N}=40)$

\begin{tabular}{ll}
\hline & COBRA \\
\hline WCSTCA &. .124 \\
PEM & .223 \\
CPTerr & .229 \\
CPTRT & .049 \\
WFT & -.12 \\
STRT & .233 \\
STerr & -.076 \\
TMT-A & $.378^{*}$ \\
TMT-B & .224 \\
AVLTi & -.119 \\
AVLTr & -.066 \\
\hline
\end{tabular}

Abbreviations: COBRA, cognitive complaints in bipolar disorder rating assessment; WCSTCA, Wisconsin card sorting test number of categories achieved; PEM, number of perseverative errors (Milner type); CPTerr, continuous performance test errors; CPTRT, continuous performance test reaction time; WFT, word fluency test; STRT, Stroop test reaction time; STerr, Stroop test number of errors. TMT-A, trail making test numeric sequence; TMT-B, trail making test alternating numeric and alphabetic sequence; AVLTi, auditory verbal learning test instant recall; AVLTr, auditory verbal learning test delayed recall. ${ }^{*} p<.05$ (twosided). 


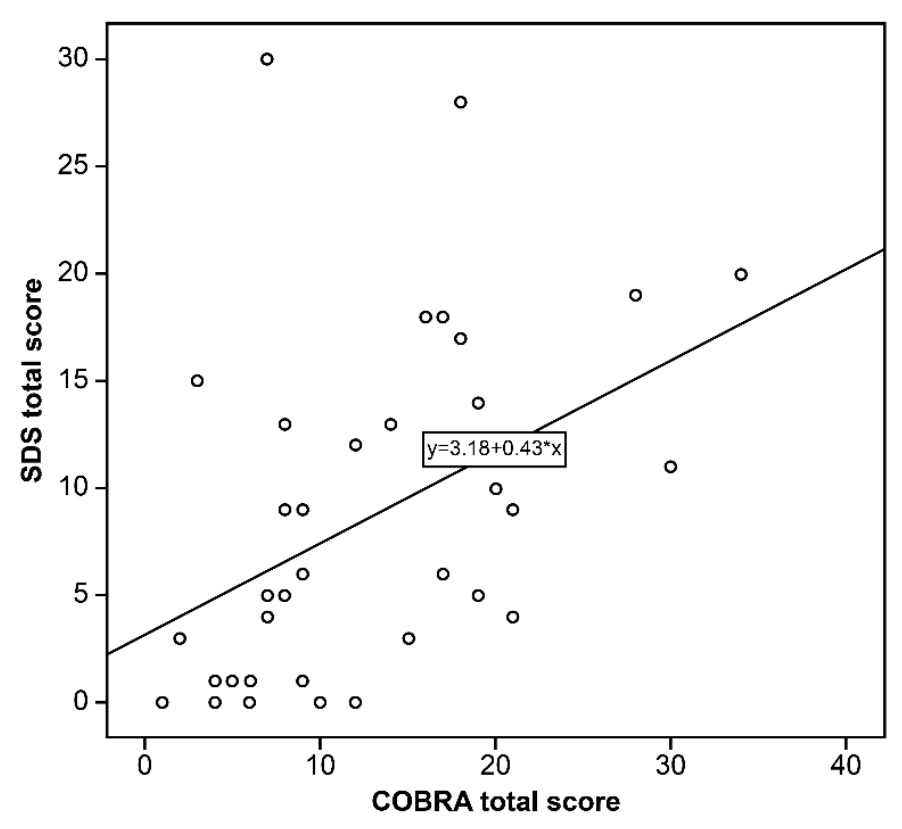




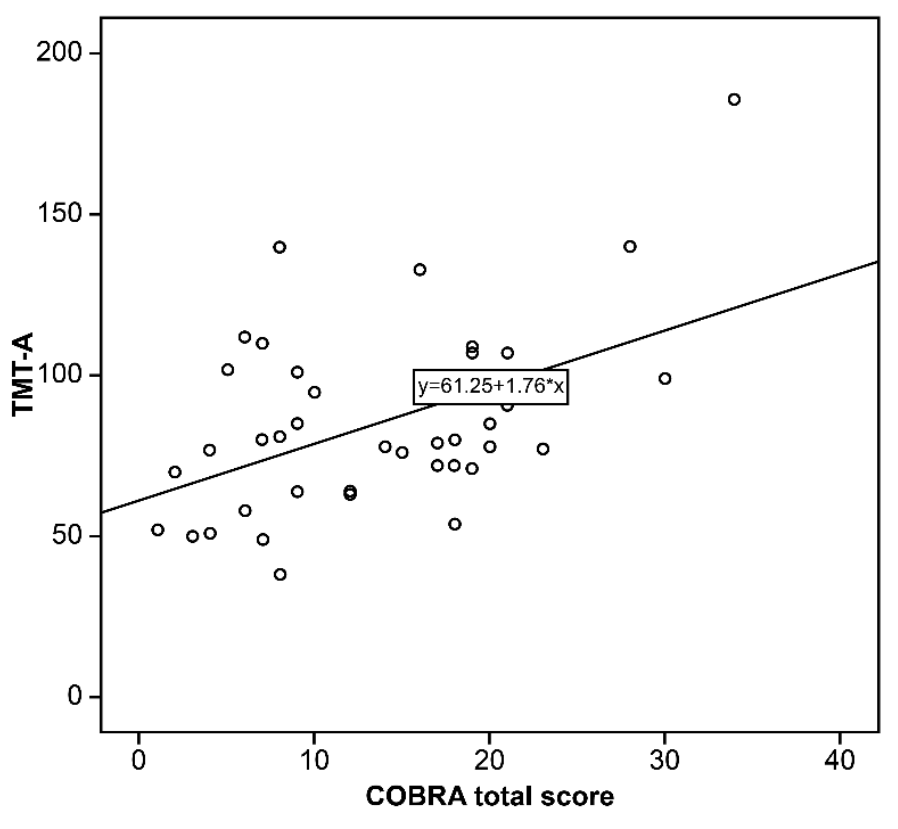

